Revista de MATEMÁticA: TeORÍA y APliCACIONEs 2016 23(1) : 291-308

CIMPA - UCR ISSN: 1409-2433 (PRINT), 2215-3373 (ONLINE)

\title{
ON A CONJECTURE OF R. BRÜCK CONCERNING MEROMORPHIC FUNCTION SHARING SMALL FUNCTIONS*
}

\author{
SOBRE UNA CONJETURA DE R. BRÜCK \\ CONCERNIENTE A LA FUNCIÓN MEROMÓRFICA \\ QUE COMPARTE PEQUEÑAS FUNCIONES
}

\author{
HONG-YAN XU ${ }^{\dagger} \quad$ CAI-FENG YI ${ }^{\ddagger} \quad$ HUA WANG ${ }^{\S}$
}

Received: 27 Apr 2015; Revised: 2 Sep 2015;

Accepted: 19 Oct 2015

\footnotetext{
${ }^{*}$ The first author was supported by the NSF of China $(11561033,11301233,11561031)$, the Natural Science Foundation of Jiangxi Province in China (20151BAB201008), and the Foundation of Education Department of Jiangxi (GJJ14644) of China.

${ }^{\dagger}$ Department of Informatics and Engineering, Jingdezhen Ceramic Institute, Jingdezhen, Jiangxi, 333403, China. E-Mail: xhyhhh@126.com

${ }^{\ddagger}$ Institute of Mathematics and informatics, Jiangxi Normal University, Nanchang, Jiangxi, 330022, China. E-Mail: yicaifeng55@163.com

${ }^{\S}$ Misma dirección que/Same address as: H.-Y.Xu. E-Mail: hhhlucy2012@126.com
} 


\begin{abstract}
In this paper, we investigate the uniqueness problem on a conjecture of R. Brück concerning meromorphic function sharing the set of small functions with its derivative, and obtain some results which improve the theorems given by Zhang and Yang.
\end{abstract}

Keywords: meromorphic function; derivative; small function.

\title{
Resumen
}

En este artículo, nvestigamos el problema de unicidad sobre una conjetura de R. Brück que concierne una función meromórfica que comparte un conjunto de pequeñas funciones con su derivada, y obtenemos algunos resultados que mejoran los teoremas dados por Zhang y Yang.

Palabras clave: función meromórfica; derivada; función pequeña.

Mathematics Subject Classification: 30D30, 30D35..

\section{Introduction and main results}

Let $f$ be a non-constant meromorphic function in the whole complex plane $\mathbb{C}$. We shall use the following standard notations of the value distribution theory:

$$
T(r, f), m(r, f), N(r, f), \bar{N}(r, f), \ldots
$$

(see Hayman [7],Yang [14] and Yi and Yang [16]). We denote by $S(r, f)$ any quantity satisfying

$$
S(r, f)=o(T(r, f)),
$$

as $r \rightarrow+\infty$, possibly outside of a set with finite measure. A meromorphic function $a$ is called a small function with respect to $f$ if $T(r, a)=S(r, f)$. Let $S(f)$ be the set of meromorphic functions in the complex plane $\mathbb{C}$ which are small functions with respect to $f$.

Let $f$ be a nonconstant meromorphic function and $a \in \widetilde{S}(f)=S(f) \cup\{\infty\}$ and $S$ be a subset of $\widetilde{S}(f)$. Define

$$
\begin{aligned}
& E(S, f)=\bigcup_{a \in S}\{z: f(z)-a=0, \quad \text { counting multiplicity }\} \\
& \bar{E}(S, f)=\bigcup_{a \in S}\{z: f(z)-a=0, \quad \text { ignoring multiplicity }\} .
\end{aligned}
$$

If $E(S, f)=E(S, g)$, we say that $f$ and $g$ share the set $S C M$; if $\bar{E}(S, f)=$ $\bar{E}(S, g)$, we say that $f$ and $g$ share the set $S I M$. Especially, let $S=\{a\}$, we 
say that $f$ and $g$ share the value $a C M$ if $E(S, f)=E(S, g)$; and we say that $f$ and $g$ share the value $a I M$ if $\bar{E}(S, f)=\bar{E}(S, g)$ (see [7]).

The shared value problems relative to a meromorphic function $f$ and its derivative $f^{(k)}$ have been a more widely studied subtopic of the uniqueness theory of entire and meromorphic functions in the field of complex analysis (see $[5,11,19,22])$.

In 1996, the following conjecture was proposed by Brück [3]:

Conjecture 1.1 Let $f$ be a non-constant entire function. Suppose that $\rho_{1}(f)$ is not a positive integer or infinite, if $f$ and $f^{\prime}$ share one finite value a $C M$, then

$$
\frac{f^{\prime}-a}{f-a}=c
$$

for some non-zero constant $c$, where $\rho_{1}(f)$ is the first iterated order of $f$ which is defined by

$$
\rho_{1}(f)=\limsup _{r \rightarrow \infty} \frac{\log \log T(r, f)}{\log r} .
$$

In 1996, Brück [3] proved that the conjecture is true when $a=0$ or $N\left(r, 1 / f^{\prime}\right)=S(r, f)$; in 1998, Gundersen and Yang [6] proved that the conjecture is true when $f$ is of finite order (see [6]); and in 2004, Chen and Shon [4] proved that the conjecture is true for entire function of first order $\rho_{1}(f)<\frac{1}{2}$. However, the corresponding conjecture for meromorphic functions fails in general, as shown by Gundersen and Yang [6], while it remains true in the case that $N\left(r, 1 / f^{\prime}\right)=S(r, f)$, see Al-Khaladi [1].

In 2008, Yang and Zhang [15] considered the case that $F=f^{n}$, where $f$ is a non-constant meromorphic function, assuming value sharing with $F$ and $F^{\prime}$ and obtained the following results:

Theorem 1.1 (see [15, Theorem 4.4]). Let $f$ be a non-constant entire function, $n \geq 7$ be an integer. Denote $F=f^{n}$. If $F$ and $F^{\prime}$ share $1 C M$, then $F=F^{\prime}$, and $f$ assumes the form

$$
f(z)=c e^{\frac{z}{n}}
$$

where $c$ is a nonzero constant.

Theorem 1.2 (see [15, Theorem 4.3]). Let $f$ be a non-constant meromorphic function and $n \geq 12$ be an integer. Denote $F=f^{n}$. If $F$ and $F^{\prime}$ share $1 C M$, then $F=F^{\prime}$, and $f$ assumes the form

$$
f(z)=c e^{\frac{z}{n}}
$$

where $c$ is a nonzero constant. 
In 2009, Zhang and Yang [20] greatly improved Theorems 1 and 2 by obtaining the following results.

Theorem 1.3 (see [20, Theorem 1.1]). Let $f$ be a non-constant entire function, $n, k$ be positive integers and $a(z)$ be a small meromorphic function of $f$ such that $a(z) \not \equiv 0, \infty$. If $f^{n}-a$ and $\left(f^{n}\right)^{(k)}-a$ share the value $0 C M$ and $n>k+1$, then $f^{n}=\left(f^{n}\right)^{(k)}$, and $f$ assumes the form

$$
f(z)=c e^{\frac{\lambda}{n} z}
$$

where $c$ is a nonzero constant and $\lambda^{k}=1$.

Theorem 1.4 (see [20, Theorem 1.2]). Let $f$ be a non-constant meromorphic function, $n, k$ be positive integers and $a(z)$ be a small meromorphic function of $f$ such that $a(z) \not \equiv 0, \infty$. If $f^{n}-a$ and $\left(f^{n}\right)^{(k)}-a$ share the value $O C M$ and $n>k+1+\sqrt{k+1}$, then $f^{n}=\left(f^{n}\right)^{(k)}$, and $f$ assumes the form

$$
f(z)=c e^{\frac{\lambda}{n} z},
$$

where $c$ is a nonzero constant and $\lambda^{k}=1$.

Remark 1.1 If $k=1$ in Theorems 1.3 and 1.4, then $n>k+1$ and $n>$ $k+1+\sqrt{k+1}$ should be replaced by $n \geq 3$ and $n \geq 4$, respectively. Thus, Theorems 1.1 and 1.2 are greatly improved by Theorems 1.3 and 1.4.

Regarding Theorems 1.1-1.4, it is natural to ask the following questions:

Question 1.1 Can the nature of sharing 1 and $a(z) C M$ be further relaxed in Theorems 1.1 and 1.3 ?

Question 1.2 What will happen when 1 and $a(z)$ are replaced by a set $S_{m}=$ $\{a(z), a(z) \omega$

$\left.\ldots, a(z) \omega^{m-1}\right\}$ in Theorems 1.1-1.4, where $\omega=\cos \frac{2 \pi}{m}+i \sin \frac{2 \pi}{m}$ and $m$ is $a$ positive integer?

In 2001, an idea of gradation of sharing of values and sets known as weighted sharing was introduced in $[9,10]$ which measures how close a shared value is to being shared $I M$ or to being shared $C M$.

Recently, many mathematicians researched the problem on meromorphic and entire function sharing small function with its derivative by using the weighted shared idea (see $[2,8,12,13,19]$ ).

First, the notions of weighted sharing of values are introduced as follows. 
Definition $1.1([9,10])$. Let l be a nonnegative integer or infinity. For a $\in \widetilde{\mathbb{C}}$, we denote by $E_{l}(a, f)$ the set of all a-points of $f$ where an a-point of multiplicity $m$ is counted $m$ times if $m \leq l$ and $l+1$ times if $m>l$. If $E_{l}(a, f)=E_{l}(a, g)$, we say that $f, g$ share the value a with weight $l$.

We write $f, g$ share $(a, l)$ to mean that $f, g$ share the value a with weight $l$, clearly if $f, g$ share $(a, l)$, then $f, g$ share $(a, p)$ for all integer $p(0 \leq p \leq l)$. Also, we note that $f, g$ share a value a IM or CM if and only if share $(a, 0)$ or $(a, \infty)$, respectively.

Remark 1.2 Let $S$ be a subset of $\widetilde{S}(f)$, we can get the definitions of $E_{l}(S, f)$ and $E_{l}(S, f)=E_{l}(S, g)$, similarly.

With the idea of weighted sharing of values, the solution of the above questions was investigated and obtained the following results.

Theorem 1.5 Let $f$ be a non-constant entire function, $n, k, l, m$ be positive integers and $a(z)$ be a small meromorphic function of $f$ such that $a(z) \not \equiv 0, \infty$. If $E_{l}\left(S_{m}, f^{n}\right)=E_{l}\left(S_{m},\left(f^{n}\right)^{(k)}\right)$ and

$$
n>\max \left\{k+1, k+\frac{\gamma}{l m}\right\}
$$

where $\gamma=k+l+2$, then $f^{n}=\left(f^{n}\right)^{(k)}$, and $f$ assumes the form

$$
f(z)=c e^{\frac{\mu}{n} z},
$$

where $c$ is a nonzero constant and $\mu^{k m}=1$.

Theorem 1.6 Let $f$ be a non-constant meromorphic function, $n, k, l, m$ be positive integers and $a(z)$ be a small meromorphic function of $f$ such that $a(z) \not \equiv$ $0, \infty$. If $E_{l}\left(S_{m}, f^{n}\right)=E_{l}\left(S_{m},\left(f^{n}\right)^{(k)}\right)$ and

$$
n>\max \left\{k+1, \frac{l(m+1) k+2 \gamma}{2 l m}+\frac{\sqrt{4 \gamma(\gamma+l k)+(m-1)^{2} l^{2} k^{2}}}{2 l m}\right\},
$$

where $\gamma=k+l+2$. Then $f^{n}=\left(f^{n}\right)^{(k)}$, and $f$ assumes the form

$$
f(z)=c e^{\frac{\mu}{n} z},
$$

where $c$ is a nonzero constant and $\mu^{k m}=1$.

Remark 1.3 We can get Theorem 1.3 and Theorem 1.4 when $m=1, l=\infty$ in Theorem 1.5 and Theorem 1.6, respectively. 
Corollary 1.1 Let $f$ be a non-constant entire function, $n, l(\geq 4)$ be positive integers and $a(z)$ be a small meromorphic function of $f$ such that $a(z) \not \equiv 0, \infty$. Denote $F=f^{n}$, if $E_{l}(a, F)=E_{l}\left(a, F^{\prime}\right)$ and $n \geq 3$, then $F=F^{\prime}$, and $f$ assumes the form

$$
f(z)=c e^{\frac{1}{n} z}
$$

where $c$ is a nonzero constant.

Corollary 1.2 Let $f$ be a non-constant meromorphic function, $n, l(\geq 11)$ be positive integers and $a(z)$ be a small meromorphic function of $f$ such that $a(z) \not \equiv$ $0, \infty$. Denote $F=f^{n}$, if $E_{l}(a, F)=E_{l}\left(a, F^{\prime}\right)$ and $n \geq 4$, then $F=F^{\prime}$, and $f$ assumes the form

$$
f(z)=c e^{\frac{1}{n} z}
$$

where $c$ is a nonzero constant.

Corollary 1.3 Let $f$ be a non-constant entire function, $n, l(\geq 2)$ be positive integers and $a(z)$ be a small meromorphic function of $f$ such that $a(z) \not \equiv 0, \infty$. Denote $F=f^{n}$ and $S=\{a(z),-a(z)\}$. If $E_{l}(S, F)=E_{l}\left(S, F^{\prime}\right)$ and $n \geq 3$, then $F=F^{\prime}$, and $f$ assumes the form

$$
f(z)=c e^{\frac{1}{n} z} \quad \text { or } \quad f(z)=c e^{-\frac{1}{n} z}
$$

where $c$ is a nonzero constant.

Corollary 1.4 Let $f$ be a non-constant entire function, $n, l, k(\geq 1)$ be positive integers and $a(z)$ be a small meromorphic function of $f$ such that $a(z) \not \equiv 0, \infty$. Denote $F=f^{n}$ and $S=\{a(z),-a(z)\}$. If $E_{l}(S, F)=E_{l}\left(S, F^{(k)}\right)$ and $n \geq \max \{3, k+1\}$ and $l \geq k+2$, then $F=F^{(k)}$, and $f$ assumes the form

$$
f(z)=c e^{\frac{\lambda}{n} z}
$$

where $c$ is a nonzero constant and $\lambda^{k}= \pm 1$.

Corollary 1.5 Let $f$ be a non-constant meromorphic function, $n, l(\geq 4)$ be positive integers and $a(z)$ be a small meromorphic function of $f$ such that $a(z) \not \equiv$ $0, \infty$. Denote $F=f^{n}$ and $S=\{a(z),-a(z)\}$. If $E_{l}(S, F)=E_{l}\left(S, F^{\prime}\right)$ and $n \geq 3$, then $F=F^{\prime}$, f assumes the form

$$
f(z)=c e^{\frac{1}{n} z} \quad \text { or } \quad f(z)=c e^{-\frac{1}{n} z}
$$

where $c$ is a nonzero constant. 
Remark 1.4 Obviously, Corollary 1.1 and Corollary 1.2 improve Theorem 1.1 and Theorem 1.2, respectively.

Though the standard definitions and notations of the value distribution theory are available in [7,14], we explain the following definition and notations which is used in the paper.

Definition 1.2 (see [2,17]). When $f$ and $g$ share 1 IM, we denote by $N_{L}(r, 1 ; f)$ the counting function of the 1-points of $f$ whose multiplicities are greater than 1-points of $g$; Similarly, we have $N_{L}(r, 1 ; g)$. Let $z_{0}$ be a zero of $f-1$ of multiplicity $p$ and a zero of $g-1$ of multiplicity $q$, we also denote by $N_{11}(r, 1 ; f)$ the counting function of those 1-points of $f$ where $p=q=1 ; \bar{N}_{E}^{(2}(r, 1 ; f)$ denotes the counting function of those 1-points of $f$ where $p=q \geq 2$, each point in these counting functions is counted only once. In the same way, one can define $N_{11}(r, 1 ; g), \bar{N}_{E}^{(2}(r, 1 ; g)$.

\section{Some lemmas}

For $a \in \widetilde{\mathbb{C}}$ and $l$ a positive integer, let $f$ be a non-constant meromorphic function, we denote by $N(r, a ; f \mid=1)$ the counting function of simple $a$-points of $f$, denote by $N(r, a ; f \mid \leq l)(N(r, a ; f \mid \geq l))$ the counting functions of those $a$-points of $f$ whose multiplicities are not greater (less) than $l$ where each $a$-point is counted according to its multiplicity(see [7]). $\bar{N}(r, a ; f \mid \leq l)(\bar{N}(r, a ; f \mid \geq l))$ are defined similarly, where in counting the $a$-points of $f$ we ignore the multiplicities. And set $N_{l}(r, a ; f)=\bar{N}(r, a ; f)+\bar{N}(r, a ; f \mid \geq 2)+\cdots+\bar{N}(r, a ; f \mid \geq l)$. In this paper, we will use the notations as follows

$$
\begin{aligned}
& H=\left(\frac{F^{\prime \prime}}{F^{\prime}}-\frac{2 F^{\prime}}{F-1}\right)-\left(\frac{G^{\prime \prime}}{G^{\prime}}-\frac{2 G^{\prime}}{G-1}\right), \\
& V=\left(\frac{F^{\prime}}{F-1}-\frac{F^{\prime}}{F}\right)-\left(\frac{G^{\prime}}{G-1}-\frac{G^{\prime}}{G}\right),
\end{aligned}
$$

and

$$
U=\frac{F^{\prime}}{F-1}-\frac{G^{\prime}}{G-1} .
$$

For the proof of our results we need the following lemmas.

Lemma 2.1 (see [16, Page 27, Theorem 1.12]). Let $f$ be a nonconstant meromorphic function and $P(f)=a_{0}+a_{1} f+a_{2} f^{2}+\cdots+a_{n} f^{n}$, where $a_{0}, a_{1}, a_{2}$, $\cdots, a_{n}$ are constants and $a_{n} \neq 0$. Then

$$
T(r, P(f))=n T(r, f)+S(r, f) .
$$


Lemma 2.2 (see [18, Lemma 3]). Let $H$ be given by (3), $F$ and $G$ be two nonconstant meromorphic functions. If $H \neq 0$, then

$$
N_{11}(r, 1 ; F) \leq N(r, H)+S(r, F)+S(r, G) .
$$

Lemma 2.3 (see [21, Lemma 3]). Suppose that $f$ is a nonconstant meromorphic function and $k, p$ are positive integers. Then

$$
\begin{gathered}
N_{p}\left(r, 0 ; f^{(k)}\right) \leq T\left(r, f^{(k)}\right)-T(r, f)+N_{p+k}(r, 0 ; f)+S(r, f), \\
N_{p}\left(r, 0 ; f^{(k)}\right) \leq k \bar{N}(r, f)+N_{p+k}(r, 0 ; f)+S(r, f) .
\end{gathered}
$$

Lemma 2.4 Let $f$ be a non-constant meromorphic function, $n, k, m$ be positive integers and $a(z)$ be a small meromorphic functions of $f$ such that $a(z) \not \equiv 0, \infty$, and let $F_{1}=\frac{f^{n}}{a}, G_{1}=\frac{\left(f^{n}\right)^{(k)}}{a}$. If $f^{n}$ and $\left(f^{n}\right)^{(k)}$ share $S_{m} I M$ and $n>k+1$, and if $H \neq 0$, then

$$
T(r, f)=O(\bar{N}(r, f)+\bar{N}(r, 0 ; f)),
$$

where $H$ is given by (3), and $F=\left(F_{1}\right)^{m}, G=\left(G_{1}\right)^{m}$.

Proof: Since $f^{n},\left(f^{n}\right)^{(k)}$ share $S_{m} I M$ and $F_{1}=\frac{f^{n}}{a}, G_{1}=\frac{\left(f^{n}\right)^{(k)}}{a}$, then $F, G$ share $1 I M$ with the possible exception of the zeros and poles of $a(z)$. By using the same argument as in Lemma 2.3 of [20], and since $F_{1}=\frac{f^{n}}{a}, G_{1}=\frac{\left(f^{n}\right)^{(k)}}{a}$, it follows from Lemma 2.3 that

$$
\begin{aligned}
T\left(r,\left(G_{1}\right)^{m}\right) \leq & N_{2}\left(r, 0 ;\left(F_{1}\right)^{m}\right)+N_{2}\left(r, 0 ;\left(G_{1}\right)^{m}\right)+3 \bar{N}\left(r,\left(F_{1}\right)^{m}\right) \\
& +N_{L}\left(r, 1 ;\left(F_{1}\right)^{m}\right)+2 N_{L}\left(r, 1 ;\left(G_{1}\right)^{m}\right)+S(r, f) \\
\leq & N_{2}\left(r, 0 ; f^{n}\right)+m N_{2}\left(r, 0 ;\left(f^{n}\right)^{(k)}\right)+3 \bar{N}(r, f) \\
& +N_{L}\left(r, 1 ;\left(F_{1}\right)^{m}\right)+2 N_{L}\left(r, 1 ;\left(G_{1}\right)^{m}\right)+S(r, f) \\
\leq & N_{2}\left(r, 0 ; f^{n}\right)+m N_{2+k}\left(r, 0 ; f^{n}\right)+m T\left(r,\left(f^{n}\right)^{(k)}\right)-m T\left(r, f^{n}\right) \\
& +3 \bar{N}(r, f)+N_{L}\left(r, 1 ;\left(F_{1}\right)^{m}\right)+2 N_{L}\left(r, 1 ;\left(G_{1}\right)^{m}\right)+S(r, f) .
\end{aligned}
$$

Since $m T\left(r,\left(f^{n}\right)^{(k)}\right) \leq T\left(r,\left(G_{1}\right)^{m}\right)+S(r, f)$, it follows from (6) that

$$
\begin{aligned}
m T\left(r, f^{n}\right) \leq & 2 \bar{N}(r, 0 ; f)+m N_{2+k}\left(r, 0 ; f^{n}\right)+3 \bar{N}(r, f) \\
& +N_{L}\left(r, 1 ;\left(F_{1}\right)^{m}\right)+2 N_{L}\left(r, 1 ;\left(G_{1}\right)^{m}\right)+S(r, f) .
\end{aligned}
$$


Since $n>k+1$ and $F=\left(F_{1}\right)^{m}, G=\left(G_{1}\right)^{m}$, and by Lemma 2.3, we have

$$
\begin{aligned}
N_{L}(r, 1 ; F) & \leq N\left(r, \frac{F}{F^{\prime}}\right) \leq N\left(r, \frac{F^{\prime}}{F}\right)+S(r, f) \\
& \leq \bar{N}\left(r, 0 ; F_{1}\right)+\bar{N}\left(r, F_{1}\right)+S(r, f) \\
& \leq \bar{N}(r, 0 ; f)+\bar{N}(r, f)+S(r, f),
\end{aligned}
$$

and

$$
\begin{aligned}
N_{L}(r, 1 ; G) & \leq N\left(r, \frac{G}{G^{\prime}}\right) \leq N\left(r, \frac{G^{\prime}}{G}\right)+S(r, f) \\
& \leq \bar{N}\left(r, 0 ; G_{1}\right)+\bar{N}\left(r, G_{1}\right)+S(r, f) \\
& \leq N_{1}\left(r, 0 ;\left(f^{n}\right)^{(k)}\right)+\bar{N}(r, f)+S(r, f) \\
& \leq N_{1+k}\left(r, 0 ; f^{n}\right)+(k+1) \bar{N}(r, f)+S(r, f) \\
& \leq(k+1) \bar{N}(r, 0 ; f)+(k+1) \bar{N}(r, f)+S(r, f) .
\end{aligned}
$$

Substituting (8) and (9) to (7), by Lemmas 2.1 and 2.3, we have

$$
\begin{aligned}
T(r, f) \leq & \frac{(m+2) k+2 m+5}{m n} \bar{N}(r, 0 ; f)+\frac{2 k+6}{m n} \bar{N}(r, f) \\
& +S(r, f) .
\end{aligned}
$$

Thus, the proof of Lemma 2.4 is completed.

Lemma 2.5 Let $V$ be given by (4), and $F, G, F_{1}, G_{1}$ be given by Lemma 2.4. If $n, m, k$ are positive integers such that $n>k+1$, and $V=0$, then

$$
f^{n}=t\left(f^{n}\right)^{(k)}
$$

where $t^{m}=1$, and $f$ assumes the form

$$
f(z)=c e^{\frac{\mu}{n} z},
$$

where $c$ is a non-zero constant and $\mu^{m k}=1$.

Proof: From $V=0$ and the definitions of $F, G$, we get

$$
1-\frac{1}{\left(F_{1}\right)^{m}}=A-\frac{A}{\left(G_{1}\right)^{m}}
$$

where $A$ is a non-zero constant. We consider two cases as follows. 
Case 1. $N(r, f)=S(r, f)$. If $A \neq 1$, from (11) we have $\bar{N}\left(r, \frac{1}{1-A} ;\left(F_{1}\right)^{m}\right)=$ $\bar{N}\left(r,\left(G_{1}\right)^{m}\right)=S(r, f)$. By the second fundamental theorem and the definitions of $F_{1}, G_{1}$, we have

$$
\begin{aligned}
n m T(r, f) \leq & T\left(r,\left(F_{1}\right)^{m}\right) \leq \bar{N}\left(r,\left(F_{1}\right)^{m}\right)+\bar{N}\left(r, 0 ;\left(F_{1}\right)^{m}\right) \\
& +\bar{N}\left(r, \frac{1}{1-A} ;\left(F_{1}\right)^{m}\right)+S(r, f) \\
\leq & \bar{N}(r, 0 ; f)+S(r, f) .
\end{aligned}
$$

Thus, we get a contradiction. Therefore $A=1$.

Case 2. $N(r, f) \neq S(r, f)$. Then there exists a $z_{0}$ which is not a zero or pole of $a(z)$ such that $\frac{1}{f\left(z_{0}\right)}=0$, so $\frac{1}{\left(F_{1}\left(z_{0}\right)\right)^{m}}=\frac{1}{\left(G_{1}\left(z_{0}\right)\right)^{m}}=0$. Therefore, from (11) we get $A=1$.

Thus, by (11) and $A=1$, then $\left(F_{1}\right)^{m}=\left(G_{1}\right)^{m}$, that is,

$$
f^{n}=t\left(f^{n}\right)^{(k)},
$$

where $t^{m}=1$.

By (12) and $n>k+1$, if $z_{0}$ is a zero of $f$ with the multiplicity $p$, then $z_{0}$ is a zero of $f^{n}$ with the multiplicity $n p$ and a zero of $\left(f^{n}\right)^{(k)}$ with the multiplicity $n p-k$, which is impossible. Then we get that 0 is a Picard exceptional value of $f$. From (12), we have

$$
f(z)=c e^{\frac{\mu}{n} z},
$$

where $c$ is a non-zero constant and $\mu^{m k}=1$.

Thus, the proof of Lemma 2.5 is completed.

Lemma 2.6 Let $V$ be given by (4), and $F, G, F_{1}, G_{1}$ be given by Lemma 2.4, $n, m, k$ be positive integers. If $V \neq 0$, then

$$
(m n-1) \bar{N}(r, f) \leq N(r, V)+S(r, f) .
$$

Proof: From (4) and the definitions of $F, G$, we see that if $z_{0}$ is a pole of $f$ with the multiplicity $p$ such that $a\left(z_{0}\right) \neq 0$ and $a\left(z_{0}\right) \neq \infty$, then $z_{0}$ is a zero of $\frac{F^{\prime}}{F-1}-\frac{F^{\prime}}{F}$ with the multiplicity $m n p-1$ and a zero of $\frac{G^{\prime}}{G-1}-\frac{G^{\prime}}{G}$ with the multiplicity $m(n p+k)-1$. Therefore, $z_{0}$ is zero of $V$ with the multiplicity $q \geq m n-1$. Noting the $m(r, V)=S(r, f)$ and from (4), we have

$$
\begin{aligned}
(m n-1) \bar{N}(r, f) & \leq N(r, 0 ; V)+S(r, f) \leq T(r, V)+S(r, f) \\
& \leq N(r, V)+S(r, f) .
\end{aligned}
$$


Lemma 2.7 Let $U$ be given by (5), and $F, G, F_{1}, G_{1}$ be given by Lemma 2.4. If $n, m, k$ are positive integers such that $n>k+1$, and $U=0$, then

$$
f^{n}=t\left(f^{n}\right)^{(k)}
$$

where $t^{m}=1$, and $f$ assumes the form

$$
f(z)=c e^{\frac{\mu}{n} z},
$$

where $c$ is a non-zero constant and $\mu^{m k}=1$.

Proof: From $U=0$, we get

$$
F=B G+1-B
$$

where $B$ is a non-zero constant. By the definitions of $F, G, F_{1}, G_{1}$, we get that $N(r, f)=S(r, f)$. We discuss the following two cases:

Case 1. $B=1$. Then $F=G$, that is, $\left(F_{1}\right)^{m}=\left(G_{1}\right)^{m}$. Hence we have

$$
f^{n}=t\left(f^{n}\right)^{(k)},
$$

where $t^{m}=1$. By Lemma 2.5, we can get the conclusions of Lemma 2.7.

Case 2. $B \neq 1$. If $N(r, 0 ; f) \neq S(r, f)$, then there exists a point $z_{0}$ such that $f\left(z_{0}\right)=0$ and $a\left(z_{0}\right) \neq 0$. Since $n>k+1$, we have $F\left(z_{0}\right)=G\left(z_{0}\right)=0$. From (14), we get $B=1$, a contradiction.

If $N(r, 0 ; f)=S(r, f)$, then from (14), we get

$$
\begin{aligned}
\bar{N}(r, 1-B ; F) & =\bar{N}(r, 0 ; G) \\
& \leq(k+1) \bar{N}(r, 0 ; f)+k \bar{N}(r, f) \\
& =S(r, f) .
\end{aligned}
$$

By the second fundamental theorem and $N(r, 0 ; f)=N(r, f)=S(r, f)$, we have

$$
\begin{aligned}
m n T(r, f) & \leq T(r, F)+S(r, f) \\
& \leq \bar{N}(r, F)+\bar{N}(r, 0 ; F)+\bar{N}(r, 1-B ; F)+S(r, f) \\
& \leq \bar{N}(r, 0 ; f)+\bar{N}(r, f)+\bar{N}(r, 0 ; G)+S(r, f) \\
& \leq S(r, f) .
\end{aligned}
$$

Since $n, m$ are positive integers, we get another contradiction.

Thus, the proof of Lemma 2.7 is completed. 
Lemma 2.8 Let $U$ be given by (5), and $F, G, F_{1}, G_{1}$ be given by Lemma 2.4. If $n, m, k$ are positive integers such that $n>k+1$, and $U \neq 0$, then

$$
[m(n-k)-1] \bar{N}(r, 0 ; f) \leq N(r, U)+S(r, f) .
$$

Proof: We propose to follow the idea in the proof of Lemma 2.8 in [20]. If $z_{0}$ is a zero of $f$ with the multiplicity $p(\geq 1)$ such that $a\left(z_{0}\right) \neq 0, \infty$. From the definitions of $F, G, F_{1}, G_{1}$, we get that $z_{0}$ is a zero of $\frac{F^{\prime}}{F-1}$ with the multiplicity $m n p-1$ and a zero of $\frac{G^{\prime}}{G-1}$ with the multiplicity $m(n p-k)-1$. Thus, $z_{0}$ is a zero of $U$ with the multiplicity at least $m(n-k)-1$. Since $m(r, U)=S(r, f)$, we have

$$
\begin{aligned}
{[m(n-k)-1] \bar{N}(r, 0 ; f) } & \leq N(r, 0 ; U)+S(r, f) \leq T(r, U)+S(r, f) \\
& \leq N(r, U)+S(r, f) .
\end{aligned}
$$

Thus, the proof of Lemma 2.8 is completed.

Lemma 2.9 Let $H$ be given by (3), and $F, G, F_{1}, G_{1}$ be given by Lemma 2.4. If $n, m, k$ are positive integers such that $n>k+1$, and

$$
\bar{N}(r, f)=\bar{N}(r, 0 ; f)=S(r, f),
$$

and $H=0$, then

$$
f^{n}=t\left(f^{n}\right)^{(k)},
$$

where $t^{m}=1$, and $f$ assumes the form

$$
f(z)=c e^{\frac{\mu}{n} z},
$$

where $c$ is a non-zero constant and $\mu^{m k}=1$.

Proof: From $H=0$, by integration, from (3) we get that

$$
\frac{1}{F-1}=\frac{C}{G-1}+D
$$

where $C(\neq 0)$ and $D$ are constants. From (17) we have

$$
G=\frac{(D-C) F+(C-D-1)}{D F-(D+1)},
$$

that is,

$$
\left(G_{1}\right)^{m}=\frac{(D-C)\left(F_{1}\right)^{m}+(C-D-1)}{D\left(F_{1}\right)^{m}-(D+1)} .
$$


We consider three cases as follows.

Case 1. Suppose $D \neq 0,-1$. From (18), we have $\bar{N}\left(r, \frac{D+1}{D} ;\left(F_{1}\right)^{m}\right)=$ $\bar{N}\left(r,\left(G_{1}\right)^{m}\right)$. By the second fundamental theorem and the assumptions of Lemma 2.9 and $S(r, F)=S(r, f)$, we get

$$
\begin{aligned}
m n T(r, f) & =T\left(r,\left(F_{1}\right)^{m}\right)+S(r, f) \\
& \leq \bar{N}\left(r,\left(F_{1}\right)^{m}\right)+\bar{N}\left(r, 0 ;\left(F_{1}\right)^{m}\right)+\bar{N}\left(r, \frac{D+1}{D} ;\left(F_{1}\right)^{m}\right)+S(r, f) \\
& \leq \bar{N}(r, f)+\bar{N}(r, 0 ; f)+\bar{N}\left(r,\left(G_{1}\right)^{m}\right)+S(r, f) \\
& \leq S(r, f) .
\end{aligned}
$$

Since $n, m$ are positive integers, we get a contradiction.

Case 2. Suppose $D=0$. From (18), we have $\bar{N}\left(r, \frac{C-1}{C} ;\left(F_{1}\right)^{m}\right)=\bar{N}\left(r, 0 ;\left(G_{1}\right)^{m}\right)$. We consider two subcases as follows.

Subcase 2.1. $C \neq 1$. By the second fundamental theorem and the assumptions of Lemma 2.9 and $S(r, F)=S(r, f)$, we get

$$
\begin{aligned}
m n T(r, f) & =T\left(r,\left(F_{1}\right)^{m}\right)+S(r, f) \\
& \leq \bar{N}\left(r,\left(F_{1}\right)^{m}\right)+\bar{N}\left(r, 0 ;\left(F_{1}\right)^{m}\right)+\bar{N}\left(r, \frac{C-1}{C} ;\left(F_{1}\right)^{m}\right)+S(r, f) \\
& =\bar{N}(r, f)+\bar{N}(r, 0 ; f)+\bar{N}\left(r, 0 ; G^{m}\right)+S(r, f) \\
& \leq(k+2) \bar{N}(r, 0 ; f)+(k+1) \bar{N}(r, f)+S(r, f) \\
& \leq S(r, f) .
\end{aligned}
$$

Since $n, m$ are positive integers, we get a contradiction.

Subcase 2.2. $C=1$. Then we have $\left(F_{1}\right)^{m}=\left(G_{1}\right)^{m}$ that is, $f^{n}=t\left(f^{n}\right)^{(k)}$, where $t^{m}=1$. From the proof of Lemma 2.5, we get the conclusions of Lemma 2.9.

Case 3. $D+1=0$. From (18), we have

$$
\left(G_{1}\right)^{m}=\frac{(C+1)\left(F_{1}\right)^{m}-C}{\left(F_{1}\right)^{m}} .
$$

Employing the similar proofing of Case 2 of Lemma 2.9, we get $\left(F_{1}\right)^{m}\left(G_{1}\right)^{m} \equiv 1$, that is. $f^{n}\left(f^{n}\right)^{(k)}=t a^{2}(z)$, where $t^{m}=1$.

From $\bar{N}(r, f)=\bar{N}(r, 0 ; f)=S(r, f)$, we have

$$
\begin{aligned}
2 T\left(r, \frac{f^{n}}{a}\right) & =T\left(r, \frac{f^{2 n}}{a^{2}}\right)=T\left(r, \frac{t a^{2}}{f^{2 n}}\right)+O(1)=T\left(r, \frac{\left(f^{n}\right)^{(k)}}{f^{n}}\right)+O(1) \\
& \leq m\left(r, \frac{\left(f^{n}\right)^{(k)}}{f^{n}}\right)+N\left(r, \frac{\left(f^{n}\right)^{(k)}}{f^{n}}\right)+O(1) \\
& \leq(k+1) \bar{N}(r, f)+\bar{N}(r, 0 ; f)+O(1) \\
& \leq S(r, f) .
\end{aligned}
$$

Therefore, we have $T(r, f)=S(r, f)$, a contradiction.

Thus, the proof of Lemma 2.9 is completed. 


\section{Proofs of theorems 1.5 and 1.6}

In this section, let $H, V$ and $U$ be given by (3), (4) and (5) respectively, and $F, G, F_{1}, G_{1}$ be stated as in Lemma 2.4.

\subsection{Proof of theorem 1.6}

Proof: We propose to follow the idea in [20].

If $V=0$ or $U=0$, we get the conclusions of Theorem 1.6 from Lemmas 2.5 and 2.7 .

Let $V \neq 0$ and $U \neq 0$. From the assumptions of Theorem 1.6, we get $E_{l}(1, F)=E_{l}(1, G)$. From (4) and (5), we get

$N(r, V) \leq \bar{N}(r, 0 ; G)+\bar{N}(r, 1 ; F \mid \geq l+1)+\bar{N}(r, 1 ; G \mid \geq l+1)+S(r, f)$,

and

$$
N(r, U) \leq \bar{N}(r, f)+\bar{N}(r, 1 ; F \mid \geq l+1)+\bar{N}(r, 1 ; G \mid \geq l+1)+S(r, f) .
$$

Since

$$
\begin{aligned}
\bar{N}(r, 1 ; F \mid \geq l+1) & \leq \frac{1}{l} N\left(r, \frac{F}{F^{\prime}}\right) \leq \frac{1}{l} N\left(r, \frac{F^{\prime}}{F}\right)+S(r, f) \\
& \leq \frac{1}{l} \bar{N}(r, 0 ; F)+\frac{1}{l} \bar{N}(r, F)+S(r, f) \\
& \leq \frac{1}{l} \bar{N}(r, 0 ; f)+\frac{1}{l} \bar{N}(r, f)+S(r, f),
\end{aligned}
$$

and

$$
\begin{aligned}
\bar{N}(r, 1 ; G \mid \geq l+1) & \leq \frac{1}{l} N\left(r, \frac{G}{G^{\prime}}\right) \leq \frac{1}{l} N\left(r, \frac{G^{\prime}}{G}\right)+S(r, f) \\
& \leq \frac{1}{l} \bar{N}(r, 0 ; G)+\frac{1}{l} \bar{N}(r, G)+S(r, f) \\
& \leq \frac{k+1}{l} \bar{N}(r, 0 ; f)+\frac{k+1}{l} \bar{N}(r, f)+S(r, f) .
\end{aligned}
$$

From (19)-(22), we have

$$
N(r, V) \leq \frac{l k+\gamma}{l} \bar{N}(r, 0 ; f)+\frac{l k+k+2}{l} \bar{N}(r, f)+S(r, f),
$$

and

$$
N(r, U) \leq \frac{k+2}{l} \bar{N}(r, 0 ; f)+\frac{\gamma}{l} \bar{N}(r, f)+S(r, f),
$$

where $\gamma=k+l+2$. 
From (23), (24) and Lemmas 2.6 and 2.8, we have

$$
[l m n-(l k+\gamma)] \bar{N}(r, f) \leq(l k+\gamma) \bar{N}(r, 0 ; f)+S(r, f),
$$

and

$$
[\operatorname{lm}(n-k)-\gamma] \bar{N}(r, 0 ; f) \leq \gamma \bar{N}(r, f)+S(r, f) .
$$

From (25) and (26), we have

$$
\{[l m n-(l k+\gamma)][\operatorname{lm}(n-k)-\gamma]-(l k+\gamma) \gamma\} \bar{N}(r, f) \leq S(r, f),
$$

and

$$
\{[l m n-(l k+\gamma)][\operatorname{lm}(n-k)-\gamma]-(l k+\gamma) \gamma\} \bar{N}(r, 0 ; f) \leq S(r, f) .
$$

From (2), we have $[\operatorname{lmn}-(l k+\gamma)][\operatorname{lm}(n-k)-\gamma]-(l k+\gamma) \gamma>0$. Thus, we get

$$
\bar{N}(r, f)=\bar{N}(r, 0 ; f)=S(r, f) .
$$

Next, two cases are considered as follows.

Case 1. $H \neq 0$. From Lemma 2.4 and (27), we get $T(r, f)=S(r, f)$, a contradiction.

Case 2. $H=0$. From Lemma 2.9, we get the conclusions of Theorem 1.6.

Therefore, the proof of Theorem 1.6 is completed.

\subsection{Proof of theorem 1.5}

Proof: Since $f$ is an entire function, we have $\bar{N}(r, f)=S(r, f)$. If $U=0$, we can get the conclusion of Theorem 1.5 from Lemma 2.7. If $U \neq 0$, then from Lemma 2.8 and (24) we have

$$
[\operatorname{lm}(n-k)-\gamma] \bar{N}(r, 0 ; f) \leq S(r, f) .
$$

From (1), we get $l m(n-k)-\gamma>0$. Thus, we have $\bar{N}(r, 0 ; f)=S(r, f)$ from (28). By using the same argument as in Cases 1 and 2 of the proof of Theorem 1.6, we get the conclusions of Theorem 1.5.

Therefore, the proof of Theorem 1.5 is completed.

\section{Proofs of corollaries 1.1, 1.3 and 1.4}

\subsection{Proof of corollary 1.1}

Proof: If $k=1, m=1$ and $l \geq 4$, then we have $\gamma=l+3$ and $k+\frac{\gamma}{l}=2+\frac{3}{l}<3$. Thus, we get the conclusions of Corollary 1.1 from Theorem 1.5. 


\subsection{Proof of corollary $\mathbf{1 . 3}$}

Proof: If $k=1, m=2$ and $l \geq 2$, then we have $\gamma=l+3$ and $k+\frac{\gamma}{l}=2+\frac{3}{l}<3$. Thus, we get the conclusions of Corollary 1.3 from Theorem 1.5.

\subsection{Proof of corollary 1.4}

Proof: If $m=2$ and $l \geq k+2$, then we have $k+\frac{\gamma}{l m}=k+\frac{k+l+2}{2 l} \leq k+1$. Thus, the conclusions of Corollary 1.4 are proved from Theorem 1.5.

\section{$5 \quad$ Proofs of Corollaries 1.2 and 1.5}

\subsection{Proof of Corollary 1.2}

Proof: If $k=1, m=1$, then we have

$$
\begin{aligned}
& \frac{l(m+1) k+2 \gamma}{2 l m}+\frac{\sqrt{4 \gamma(\gamma+l k)+(m-1)^{2} l^{2} k^{2}}}{2 l m} \\
= & 2+\frac{3}{l}+\frac{\sqrt{(2 l+3)(l+3)}}{l} .
\end{aligned}
$$

Since $l \geq 11$, we get

$$
k+1=2<2+\frac{3}{l}+\frac{\sqrt{(2 l+3)(l+3)}}{l}<4 .
$$

Thus, the conclusions of Corollary 1.2 are proved from (2), (30) and Theorem 1.6.

\subsection{Proof of corollary 1.5}

Proof: If $k=1, m=2$, then we have

$$
\frac{l(m+1) k+2 \gamma}{2 l m}+\frac{\sqrt{4 \gamma(\gamma+l k)+(m-1)^{2} l^{2} k^{2}}}{2 l m}=2+\frac{3}{l} .
$$

Since $l \geq 4$, we get $2<2+\frac{3}{l}<3$. Thus, the conclusions of Corollary 1.5 are proved from (31) and Theorem 1.6. 


\section{References}

[1] Al-Khaladi, A. (2005) "On meromorphic functions taht share one value with their derivative", Analysis 25: 131-140.

[2] Banerjee, A. (2007) "Weighted sharing of a small function by a meromorphic function and its derivative", Comput. Math. Appl. 53: 1750-1761.

[3] Brück, R. (1996) "On entire functions which share one value $C M$ with their first derivative", Results Math. 30: 21-24.

[4] Chen, Z.-X.; Shon, K.H. (2004) "On conjecture of R. Brück concerning entire function sharing one value $C M$ with its derivative", Taiwanese $J$. Math. 8: 235-244.

[5] Gundersen, G.G. (1980) "Meromorphic functions that share finite values with their derivative", J. Math. Anal. Appl. 75: 441-446.

[6] Gundersen, G.G.; Yang, L.-Z. (1998) "Entire functions that share one value with one or two of their derivatives", J. Math. Anal. Appl. 223: 88-95.

[7] Hayman, W.K. (1964) Meromorphic Functions. The Clarendon Press, Oxford.

[8] Lin, W.-C.; Yi, H.-X. (2004) "Uniqueness theorems for meromorphic function”, Indian J. Pure Appl. Math. 35: 121-132.

[9] Lahiri, I. (2001) "Weighted sharing and uniqueness of meromorphic functions", Nagoya Math. J. 16(1): 193-206.

[10] Lahiri, I. (2001) "Weighted value sharing and uniqueness of meromorphic functions", Complex Var. Theory Appl. 46: 241-253.

[11] Mues, E.; Steinmetz, N. (1986) "Meromorphe funktionen, die mit ihrer ersten und zweiten Ableitung einen endlichen Wert teilen", Complex Var. Theory Appl. 6: 51-71.

[12] Xu, H.-Y. (2007) "Uniqueness of transcendental meromorphic functions with their nonlinear differential polynomials sharing the small function", Int. J. Math. Math. Sci. 2007, Article ID 84961, 14 pages.

[13] Xu, H.-Y.; Cao, T.-B. (2009) "Uniqueness of entire or meromorphic functions sharing one value or a function with finite weight", J. Inequal. Pure Appl. Math. 10, Art. 88. 
[14] Yang, L. (1993) Value Distribution Theory. Springer-Verlag, Berlin.

[15] Yang, L.-Z.; Zhang, J.-L. (2008) "Non-existence of meromorphic solutions of Fermat type functional equation", Aequationes Math. 76: 140150 .

[16] Yi, H.-X.; Yang, C.-C. (1995/2003) Uniqueness Theory of Meromorphic Functions. Science Press/Kluwer Academic Publishers, Beijing/New York.

[17] Yi, H.-X. (1999) "Meromorphic functions that share one or two values II", Kodai Math. J. 22: 264-272.

[18] Yi, H.-X. (1997) "Unicity theorems for meromorphic functions whose $n$ th derivatives share the same 1-points", Complex Var. Theory Appl. 34: 421-436.

[19] Zhang, Q.-C. (2005) "Meromorphic function that shares one small function with its derivative", J. Inequal. Pure Appl. Math. 6, Art. 116.

[20] Zhang, J.-L.; Yang, L.-Z. (2009) "A power of a meromorphic function sharing a small function with its derivative", Ann. Acad. Sci. Fenn. Math. 34: $249-260$.

[21] Zhang, J.-L.; Yang, L.-Z. (2007) "Some results related to a conjecture of R. Brück”, J. Inequal. Pure Appl. Math. 8, Art. 18.

[22] Zheng, J.-H.; Wang, S.-P. (1992) "On uncity properties of meromorphic functions and their derivatives", Adv. in Math. (in Chinese) 21: 334-341. 